\title{
Les Parcs Industriels Fournisseurs ou le choix de la proximité géographique
}

\author{
Sonia ADAM-LEDUNOIS ${ }^{1}$ \\ NIMEC - Université de Rouen \\ Jérôme GUÉDON ${ }^{2}$ \\ Laboratoire Métis - École de Management de Normandie \\ Sophie RENAULT \\ LOG - Université d'Orléans
}

La proximité géographique est régulièrement présentée comme le remède à de nombreux maux, la solution à des relations distendues, voire rompues, et cela, tant sur un plan social qu'économique. Nombre de décisions ou démarches, civiles, politiques ou stratégiques, placent ainsi la proximité au cœur des dispositifs envisagés (emplois de proximité, police de proximité, fête des voisins, pôle de compétitivité, systèmes de production localisés, districts industriels, etc.). Le mouvement amorcé dans l'industrie automobile en matière d'organisation de la production repose sur cette même logique de quête des bénéfices de la proximité. Afin d'améliorer leur compétitivité, des constructeurs automobiles ont ainsi expérimenté une forme innovante d'organisation de leur système industriel, fondée sur une localisation à proximité physique de certains de leurs équipementiers. Les Parcs Industriels Fournisseurs (PIF) matérialisent cette nouvelle organisation, réunissant à proximité géographique immédiate un constructeur et ses principaux équipementiers. Mais le rapprochement géographique d'acteurs engagés dans une même filière de production ne pourrait suffire, à lui seul, à améliorer l'efficacité d'ensemble.
Le courant de la proximité, initié par des économistes spatiaux, nuance les effets positifs du rapprochement géographique, démontrant que d'autres facteurs entrent en jeu, aujourd'hui regroupés sous le terme de proximité organisée.

Cette approche semble particulièrement intéressante et nous nous proposons d'étudier les effets de la proximité au sein des PIF, en mettant en particulier l'accent sur la coexistence de différentes formes de proximités. Notre étude a été menée au travers d'observations et d'entretiens réalisés sur trois Parcs Industriels Fournisseurs : celui de Renault Sandouville, de Renault Trucks Blainville sur Orne et de PSA-Rennes la Janais ${ }^{3}$.

Le courant de la proximité, initié par des économistes spatiaux, nuance les effets positifs du rapprochement géographique, démontrant que d'autres facteurs entrent en jeu, aujourd'hui regroupés sous le terme de proximité organisée. 


\section{La proximité : diversité et externalité}

La réussite des districts industriels italiens a souvent été expliquée par la proximité géographique des différents acteurs. Le courant de la proximité marque un tournant dans cette approche. L'originalité de ce courant réside dans la volonté de regrouper des chercheurs d'horizons disciplinaires différents, afin d'appréhender les différentes acceptions de la notion, d'identifier les stabilités qui se dessinent, ainsi que les divergences les plus importantes.

Dans un premier temps, nous affinerons la notion de proximité. Nous pourrons alors, dans un second temps, appréhender les externalités liées à la proximité.

\subsection{Les différentes formes de proximité}

La littérature distingue différentes formes de proximité ; parmi ces multiples acceptions, deux principales formes de proximité se dégagent de la littérature: la proximité géographique, relative à la notion de distance et la proximité organisée, terme proposé par Rallet et Torre (2004), que nous utiliserons dans un sens générique en référence à la capacité d'une organisation à faire interagir ses membres. L'intérêt de cette distinction réside dans l'analyse croisée de ces deux formes de proximité, afin de comprendre les interactions entre organisations et territoire.

La proximité géographique fait intuitivement référence à la notion de distance et au voisinage spatial. Mais il convient de relativiser cette notion de distance sur deux points. D'une part, la distance qui importe aujourd'hui n'est plus celle de la métrique,

On entend par proximité organisée, la capacité d'une organisation à faire interagir ses membres selon deux logiques. mais celle rapportée au temps. Une distance se mesure, pour une organisation comme pour les individus, en minutes ou en heures. Ainsi, les infrastructures de transport (ferroviaires ou routières dans la plupart des cas de proximité géographique) maillant le territoire prennent une importance particulière. D'autre part, la distance est relative à la perception de l'espace par les individus. En fonction du vécu de chacun, les temporalités liées au franchissement d'une distance n'auront pas la même incidence.

On entend par proximité organisée, la capacité d'une organisation à faire interagir ses membres selon deux logiques: une logique d'appartenance et une logique de similitude. Ces deux logiques peuvent être complémentaires, sans que cela revête un caractère obligatoire.

Selon la logique d'appartenance, les interactions sont facilitées par les règles communes qui la régissent. La logique de similitude peut se définir comme le partage par les membres d'une organisation d'un « background » commun.

Différentes analyses, en particulier celles de Rallet et Torre $^{4}$ ou de Boschma ${ }^{5}$, font référence directement ou indirectement à cette notion de proximité organisée. De manière simplifiée, il nous semble en effet que les formes de proximité proposées par Boschma (2005) offrent une déclinaison de la proximité organisée, identifiée par Rallet et Torre (2004). Si elles distinguent à l'intérieur de la proximité organisée des typologies plus ou moins détaillées, ces analyses se rejoignent en ne considérant pas comme centrale la proximité géographique, dans l'établissement de relations entre des organisations. Ces typologies insistent sur la nécessité d'intégrer, au-delà de la proximité géographique, d'autres formes de proximités afin 
d'appréender dans sa globalité le phénomène. En particulier, les auteurs mettent en exergue une forme de proximité liée, non pas à la localisation spatiale, mais à des similitudes, ressemblances et plus généralement à l'existence de points communs.

Il apparaît ainsi que les différentes formes de proximité proposées peuvent se combiner entre elles, et assurent une analyse fine des jeux de proximité susceptibles d'apparaître sur un territoire. Ainsi, la proximité physique, en multipliant les interactions entre acteurs, permet une proximité relationnelle qui prendra, selon les lieux, des formes variables. L'histoire des organisations et de leurs relations, le type d'activités, les individus qui s'inscrivent sur ces territoires et dans ces organisations singularisent la proximité relationnelle qui apparait.

Le tableau 1 met en perspective la diversité des formes de proximité ainsi que les similitudes entre les approches de Boschma ${ }^{5}$ et Rallet et Torre ${ }^{4}$.

Tableau 1 - Représentation synthétique des typologies de Boschma ${ }^{5}$ et Rallet et Torre ${ }^{4}$

\begin{tabular}{|c|c|c|}
\hline Rallet et Torre (2004) & Proximité organisée & \multirow{4}{*}{ Proximité géographique } \\
\hline \multirow{3}{*}{ Boschma (2005) } & Proximité cognitive & \multirow{3}{*}{ Proximité organisationnelle } \\
& Proximité sociale & \\
\cline { 2 - 2 } & Proximité culturelle & \\
\cline { 2 - 2 } & &
\end{tabular}

Au travers de ces deux analyses de la proximité, se dégage également l'idée d'une nuance forte du poids de la proximité géographique dans les externalités positives. La présence d'autres formes de proximités semble indispensable à l'apparition d'actifs spécifiques liés à une co-localisation d'activités. Ainsi, même si la proximité géographique prédomine notre analyse, l'ensemble des proximités envisagées constituent un système ou mix de proximité qui interagissent les unes sur les autres. Nous nous intéressons désormais à l'identification des externalités de la proximité - c'est-à-dire aux conséquences que celle-ci engendre.

\subsection{Les effets de la proximité}

S'interroger sur l'utilité du concept de proximité, dans le cadre de l'analyse des relations entre entreprises et territoire n'est pas anodin. La question de la proximité a longtemps été considérée comme un élément exclusivement bénéfique pour les organisations. Il apparaît pourtant que la proximité peut générer des externalités négatives.

\subsubsection{Les effets positifs}

La présentation des différentes formes de proximité nous amène à nous interroger sur les bénéfices de la proximité, tels qu'ils sont traités dans la littérature.

Des exemples ont montré que les relations nouées entre les individus, les entreprises, voire les institutions sur un territoire pouvaient permettre le développement de compétences spécifiques qui bénéficient naturellement à toutes les composantes inscrites sur le territoire. Ces avantages sont 
beaucoup plus difficiles à capter par des organismes situés en dehors du territoire ${ }^{6}$.

Les relations de confiance sont un élément central dans la compétitivité territoriale. Les relations de proximité permettent des engagements de nature implicite et explicite (respect de règles, contraintes de coordination respectées par tous) dont les bénéfices vont à l'ensemble des acteurs sur le territoire.

Le transfert de connaissances fait également partie des éléments clés générés par les formes de proximité 7 . Les compétences en termes d'innovation technologique ou technique, de savoir-faire, basées pour une large part sur les savoirs tacites se diffusent au sein du territoire. Ce type de mécanisme, recherché par les organisations, est caractéristique de la proximité organisée.

\subsubsection{Les effets négatifs - la proximité subie}

La proximité est considérée la plupart du temps comme un facteur positif ${ }^{8}$; pourtant, la proximité peut être vécue comme un désagrément.

La proximité vécue par l'individu peut être contradictoire. L'individu peut avoir besoin d'une proximité dans le cadre des activités économiques, mais ressentir cette proximité comme néfaste dans sa vie personnelle : bruit, pollution, loyers élevés, etc.

Cette contradiction aboutit à la définition de deux types de proximité : la proximité subie et la proximité choisie.

La proximité subie est définie, à travers la notion d'agglomération, comme un facteur économique, qui aboutit à la concentration des activités et des hommes, entraînant une dégradation des conditions de vie dans les métropoles. La proximité subie représente aux yeux de l'individu les conséquences négatives du phénomène d'agglomération. Quant à la proximité choisie, elle est le résultat des aspirations individuelles et collectives, souvent en contradiction avec les logiques économiques.

Au sein des systèmes de production localisés, la limite la plus caractéristique, est le risque d'enfermement du système sur lui-même (lock-in) et de diminution importante de sa compétitivité.

Cette approche théorique de la proximité peut à présent être confrontée à l'étude des cas de Parcs Industriels Fournisseurs du secteur automobile, la proximité étant au cœur de l'analyse des PIF.

\section{La proximité au cœur des PIF}

Le PIF est un espace réservé à quelques fournisseurs de premier plan à l'intérieur ou à proximité immédiate d'une usine de production d'un constructeur. L'essence des PIF est de rapprocher en un même lieu des acteurs indépendants, impliqués dans une même filière de production.

\subsection{La convergence des proximités}

La motivation principale prévalant à la création de PIF repose sur l'optimisation du triangle d'or qualité-coût-délai ${ }^{9}$. Cependant, au-delà de ces motivations initiales, étroitement liées à la proximité géographique, émergent progressivement d'autres formes de proximité, assimilables à la proximité organisée identifiée dans la première partie de l'article.

\subsubsection{La proximité géographique: pivot des PIF}

La création de PIF a pour objectif de réduire les coûts logistiques de transport, de gagner en réactivité, d'améliorer la qualité ou d'optimiser la «montabilité » d'un sousensemble, voire de jouer sur la somme de ces variables. En externalisant la fabrication de 
sous-ensembles, les constructeurs transmettent des maillons de leur chaîne de valeur; ils exigent de leurs fournisseurs un même service avec un coût moindre et une meilleure qualité ${ }^{9}$.

Ces modules sont volumineux et fragiles, ils doivent arriver en flux synchrones sur les chaînes de montage du constructeur, ceci dans des délais de réquisition extrêmement courts (entre une demi-heure et deux heures trente).

Le mode d'approvisionnement en flux synchrone permet de proposer au client final de très nombreuses options.

En termes de logistique, la création de PIF permet ainsi de créer le volume et la diversité au plus tard et de diminuer sensiblement le stockage. Le mode d'approvisionnement en flux synchrone permet de proposer au client final de très nombreuses options. L'existence de PIF trouve sa justification dans le nombre élevé de références produites par les fournisseurs. L'ancrage des équipementiers sur le site de production des constructeurs automobiles leur permet de réaliser au plus tard les étapes de personnalisation du produit livré - la différenciation retardée. En outre, le stockage génère de fortes contraintes financières, désormais supportées par les fournisseurs.

La qualité est ainsi optimisée, grâce aux moindres manipulations de pièces fragiles et à l'absence d'intermédiaires. Le constructeur voit ainsi dans l'implantation d'un PIF «au plus près des chaînes de montage" une sécurisation de ses approvisionnements, une réactivité accrue, un contrôle de la qualité immédiat et une possibilité de dialogue permanent avec les fournisseurs.

\subsubsection{Quand proximité géographique se} conjugue avec proximité organisée

La multiplication des interactions entre les membres d'un PIF conduit progressivement à l'homogénéisation des représentations et à l'élaboration de langages et d'approches communes. Dans le contexte des PIF, la proximité physique des établissements semble donc faciliter la diffusion de conventions communes. Les conventions spécifient les comportements acceptables et ceux qui doivent être réprimés. Elles facilitent la prise de décision en sélectionnant les attitudes convenables et établissent une hiérarchie implicite de ce qui est valorisé ou de ce qui est condamné. S'il apparaît primordial que les fournisseurs de proximité gardent leur propre identité culturelle, leur installation sur le site des constructeurs nécessite l'adoption des conventions du constructeur. Dans le cas présent, la proximité spatiale semble faciliter l'émergence d'une culture commune entre les acteurs du réseau productif. Le concept d'acculturation ${ }^{10}$ permet alors de caractériser l'ensemble des changements culturels résultant des contacts continus et directs entre les fournisseurs et leur donneur d'ordres. Une forme d'intégration culturelle est pratiquée dans les PIF; en effet, la proximité géographique entre les acteurs est à l'origine de forts liens sociaux. La démarche de création de PIF s'inscrit dans une politique partenariale impliquant des relations de confiance sur le long terme.

Les acteurs au sein des PIF sont interdépendants puisqu'ils participent à un projet productif commun. Le concept de proximité est pertinent dans l'analyse des PIF. Ainsi, la proximité organisée entre les différentes unités qui composent le réseau productif semble s'accroître grâce aux nombreuses interactions entre acteurs, favorisées par la proximité géographique. Dans le cadre des PIF, ces proximités sont 
interdépendantes dans la construction de la performance.

\subsection{VICES ET VERTUS DE LA PROXIMITÉ}

Au-delà de ces avantages initialement recherchés, d'autres effets, moins

prévisibles, peuvent être associés à la colocalisation des acteurs.

La création de PIF relève d'une décision stratégique, tant de la part des constructeurs que des équipementiers. Les différents acteurs en attendent des bénéfices tangibles, en termes d'amélioration de la qualité et de réduction des délais de livraison et par voie de conséquence de fabrication. Pourtant, au-delà de ces avantages initialement recherchés, d'autres effets, moins prévisibles, peuvent être associés à la co-localisation des acteurs. Ces résultats inattendus liés à la proximité peuvent être source d'avantages, mais présentent parfois également des inconvénients non négligeables.

\section{Au-delà de l'optimisation du triangle d'or « qualité-coûts et délai »}

Dans le contexte des PIF, l'un des effets immédiats de la proximité réside dans la création d'un espace de relations. À ce propos, nous constatons que l'ancrage territorial est facteur de coordination d'un groupe social. La bonne coordination du réseau productif repose sur un brassage constant d'informations, source d'innovation et d'avantage compétitif. Ainsi, une part importante du savoir et du savoir-faire s'acquiert plus efficacement dans le contexte réel du travail et de la production. Les communautés identifiées au cœur des PIF permettent une meilleure diffusion de l'information au sein du groupe, l'adaptation rapide au changement et une certaine forme d'innovation.
Comme nous l'avons signifié dans la première partie de cet article les relations de proximité rendent également possibles un apprentissage collectif et la circulation de connaissances non codifiables. Les relations de face-à-face semblent avoir un impact significatif sur la performance du système PIF. Les relations informelles entre les membres des PIF permettraient l'obtention d'informations stratégiques, l'établissement de relations de confiance et la mise en place d'échanges plus informels.

Par ailleurs, la proximité géographique permet l'émergence d'une plus forte confiance entre les acteurs. L'interaction entre le constructeur et ses équipementiers basés à proximité immédiate facilite non seulement la diffusion, l'acquisition et l'émergence de connaissances communes, mais également le traitement et la résolution de problèmes symptomatiques. Sur les PIF étudiés, nous avons pu relever que la proximité géographique se traduisait pour certains salariés fournisseurs, par des contacts quotidiens avec des salariés constructeurs, favorisant ainsi une proximité sociale. Cette proximité suscite le développement de procédures informelles qui permettent parfois aux acteurs d'éviter le cadre procédural en cas d'incidents qualité. En effet, le fait d'être sur place facilite l'adoption par les fournisseurs de stratégies amont de traitement des problèmes. Ils peuvent notamment récupérer les pièces mises au rebut avant qu'elles ne soient enregistrées comme défaillances fournisseuses.

L'interaction entre les firmes et les acteurs est donc facilitée par la contiguïté spatiale. Cette dernière semble alors être à l'origine du développement de ressources et compétences spécifiques.

Au-delà de ses effets positifs, la proximité peut également être à l'origine d'effets pervers... Les PIF témoignent du fait que la proximité peut être porteuse de relations 
conflictuelles et contribuer à l'apparition de tensions.

En ce qui concerne l'ensemble des acteurs impliqués, nous avons constaté la menace sociale pesant sur les PIF. En effet, le rapprochement des fournisseurs s'accompagne de mouvements de personnel, depuis les constructeurs en cas d'externalisation ou entre fournisseurs eux-mêmes. La création d'unités de proximité suscite donc la vigilance des syndicats en matière d'emploi. Ces derniers portent leur attention sur l'externalisation des activités des constructeurs, mais également vis-à-vis de l'harmonisation des conditions de travail des différentes populations. De plus, la proximité entre les unités constitutives du réseau productif laisse augurer d'une possible propagation des mouvements sociaux et d'un risque de paralysie du réseau productif. Le défaut d'équité salariale est à l'origine d'un manque d'implication du personnel et donc d'une difficulté à le fidéliser.

Du point de vue des fournisseurs, nos recherches nous ont également permis de déceler quatre principales contraintes liées à la proximité :

- Nous pouvons tout d'abord souligner le manque d'autonomie stratégique des fournisseurs qui sont «pieds et poings liés » à leur donneur d'ordres. En effet, leur activité est dédiée à leur client de proximité. Le degré de liberté du fournisseur de proximité est amoindri du fait des contraintes inhérentes à l'activité $\mathrm{du}$ constructeur (emploi du temps, volume d'activité...) ainsi que des contraintes liées au mode de réquisition en flux synchrone. La forte interdépendance des acteurs dans le cadre de l'activité productive peut alors sous de nombreux aspects devenir source de blocage.
- L'une des raisons principales à la création d'unités de proximité est la réactivité. Nous avons, à ce propos, souligné la forte sollicitation des unités de proximité. La tension des flux requiert, en effet, des réactions immédiates au moindre incident à l'origine d'un niveau de tension et de stress conséquent.

- Nous avons pu constater que certains sites avancés fournisseurs souffrent des relations instaurées avec leur maison mère. Ces dernières n'accordent pas toujours le soutien escompté par les unités implantées au sein de PIF, en proie à un stress conséquent. En effet, les constructeurs désirent un unique interlocuteur, le site avancé fournisseur ; or ce dernier ne dispose pas toujours de moyens, notamment humains, ou d'une délégation de pouvoir suffisante pour répondre aux sollicitations de son client de proximité.

- Enfin, nous avons indiqué les tentatives d'ingérence des constructeurs sur l'activité de leurs fournisseurs. Ces derniers étant installés à proximité immédiate de leur donneur d'ordres, les tentations de visites inopinées sont sur certains sites fréquentes. Ces visites peuvent relever d'une volonté d'auditer l'activité des fournisseurs dans une perspective d'amélioration continue. Mais, ces démarches interviennent également parfois suite à un incident qualité survenu sur ligne. Il s'agit alors de contrôler de façon drastique le processus des fournisseurs. Les fournisseurs souffrent donc de l'immixtion de représentants du constructeur dans leur gestion quotidienne. La dilution des frontières de la firme laisse émerger l'une des modalités de la proximité subie : la promiscuité. 
Dans certains sites étudiés, nos observations illustrent que les effets de la contiguïté notamment via l'ingérence du constructeur dans les bâtiments occupés par les fournisseurs sont une source de tension. Ainsi, la proximité géographique dont on a le plus souvent une vision positive des propriétés peut jouer un rôle conflictuel certain. Si la proximité a des effets positifs manifestes dans les PIF en termes de qualité, coût et délai, d'apprentissage, elle est à certains égards subie par les acteurs. Nombreux sont par exemple les responsables de fournisseurs de proximité indiquant que dans le cadre d'une relation qualifiée de partenariale, ils n'avaient pas le choix de l'installation à proximité. La création de PIF est à l'initiative des constructeurs. Ils souhaitent que s'implantent à proximité les fournisseurs de pièces particulièrement encombrantes, fragiles ou diversifiées. L'obtention d'un marché implique pour de tels fournisseurs qu'ils acceptent de s'implanter pour la durée du programme au sein d'un PIF. Le constructeur est lui aussi enfermé dans la relation partenariale durant la durée contractuelle. Cet état de fait suggère une ambivalence entre la proximité géographique recherchée (répondant au besoin de proximité lié notamment à la tension des flux productifs) et la proximité géographique subie (qui impose des contraintes de proximité). Par ailleurs, les contraintes liées à la tension des flux suscitent une forte relation d'interdépendance entre les acteurs. L'enfermement du réseau productif sur lui-même (lock-in) est susceptible de limiter l'accès à de nouvelles opportunités.

Nous avons d'ailleurs constaté une distanciation s'opérant entre les unités de proximité des fournisseurs présents sur le PIF et leur maison mère. Ces dernières vont jusqu'à reprocher à leurs sites avancés d'adopter la culture du client, en l'occurrence celle du constructeur.
Si nous ne pouvons nier les vertus de la proximité, elle est comme nous l'avons relevé génératrice de conflits et de tensions. Pourtant, les conflits générés par la création de PIF sont également un mode de coordination permettant l'évolution des relations interindividuelles.

La proximité observée au sein des PIF relève $\mathrm{du}$ diptyque recherché et contraint. Cela implique la quête par les acteurs d'une prompte résolution des problèmes. A titre d'exemple, la résolution d'un conflit lié à la coexistence dans un même bâtiment d'un constructeur automobile et de l'un de ses fournisseurs s'est soldée par la création d'un bâtiment plus éloigné géographiquement (même si la distance physique reste minime de l'ordre d'un kilomètre). Cela illustre le fait que les acteurs des réseaux productifs observés souhaitent bénéficier des avantages de la proximité sans en subir les inconvénients. La problématique du « proche, mais pas trop » a été à l'origine de vastes réflexions avant la création de certains parcs fournisseurs : on pouvait notamment se poser la question de la nécessité ou non d'un sas entre le site constructeur et le PIF, les questions de la cantine ou du stationnement commun entre salariés du constructeur et des fournisseurs étaient également soulevées... L'arbitrage entre une juste distance euclidienne et les conditions d'une proximité organisée est souvent à l'origine de débats à la création puis dans la gestion des PIF.

Plus globalement, dans la plupart des cas la proximité permet de dénouer les conflits qu'elle suscite! Les acteurs ont ainsi la possibilité de faire évoluer positivement les tensions via des négociations en face à face ou de manière plus technique : en élaborant des protocoles de fonctionnement commun (instauration de règles, fondant une proximité institutionnelle) facilitant l'activité productive et des relations de voisinage plus sereines. 
Les acteurs observés (constructeur et fournisseurs de proximité) ont sur certains points, en dépit de la quête d'une relation " gagnant-gagnant», des intérêts divergents. La seule proximité géographique des membres du réseau productif n'est alors pas l'unique source de conflit, la relation industrielle et conséquemment la proximité organisée font également débat.

Les nouvelles formes de coordination « interfirmes » dans l'industrie automobile sont marquées par des choix stratégiques relatifs à l'espace. La notion de proximité est au centre de l'analyse des PIF. L'intégration des fournisseurs au cœur de PIF est le reflet de l'évolution des relations dans la filière automobile et de la transformation de la nature des productions confiées aux fournisseurs. La proximité géographique permet la réduction des flux et temps de circulation des produits.
Ainsi, le rapprochement physique immédiat des équipementiers sur le site de production des constructeurs automobiles trouve notamment sa justification dans la politique du juste à temps (JAT), et dans le passage à une production modulaire. Le nécessaire accroissement des flux de matières et d'informations entre les sites contribue donc à la volonté d'agglomération des partenaires. Les différentes proximités observées au cœur du réseau productif (proximité géographique, organisée, sociale, culturelle...) permettent de dégager des externalités positives en termes d'apprentissage, de confiance, de réactivité de l'organisation productive. Pourtant, si les bénéfices de la proximité restent conséquents, il nous importait ici d'en discuter également les limites. Ainsi, notre recherche nous a permis de mettre en évidence les conflits inhérents à la proximité géographique, dimension souvent sous-estimée.

\section{Notes et références}

1 Sonia Adam-Ledunois et Sophie Renault sont respectivement maîtres de conférences en Sciences de gestion à l'université de Rouen et à l'université d'Orléans.

2 Jérôme Guédon est professeur assistant à l'Ecole de Management de Normandie.

3 Nos différentes études de cas reposent sur des séries d'entretiens menés auprès de responsables ou opérationnels des constructeurs (en particulier chargés de la mise en place du PIF, responsables logistiques, responsables achats) ainsi que de responsables de sites fournisseurs présents à proximité physique immédiate des constructeurs. Notre analyse repose sur une cinquantaine d'entretiens semi-directifs réalisés au cours de nos différentes investigations sur nos terrains d'étude.

4 Rallet A. \& Torre A. (2004), «Proximité et localisation », Économie Rurale, 280, pp. 25-41.

5 Boschma Ron A. (2005), « Proximity and innovation : a critical assessment», Regional Studies, 39, pp. 61-74.

6 Joffre P. \& Koenig G. (1992), Gestion de l'entreprise. L'entreprise, ses partenaires-adversaires, leur univers, litec.

7 Torre A. (2000), «Économie de proximité et activités agricoles et agroalimentaires : éléments d'un programme de recherche », RERU, III, pp. 407-426.

8 Torre A. (2000), «Économie de proximité et activités agricoles et agroalimentaires : éléments d'un programme de recherche », RERU, III, pp. 407-426.

9 Adam-Ledunois S. \& Renault S., (2001), « Les enjeux stratégiques de la création de parcs de fournisseurs dans l'industrie automobile », Revue Française de Gestion Industrielle, volume 20, 1/2001, pp. 5-15.

10 L'acculturation est l'ensemble des phénomènes résultant du contact direct et continu entre des groupes d'individus de cultures différentes avec des changements subséquents dans les types de culture originaux de l'un ou des autres groupes (Hersjovits \& al., 1936). 


\section{PUB}

Ministère des Affaires municipales et des Régions 